\title{
Functional and prognostic effects when emphysema complicates idiopathic pulmonary fibrosis
}

\author{
Joseph Jacob ${ }^{1}$, Brian J. Bartholmai ${ }^{2}$, Srinivasan Rajagopalan ${ }^{3}$, Maria Kokosi $^{4}$, \\ Toby M. Maher ${ }^{4}$, Arjun Nair ${ }^{1}$, Ronald Karwoski ${ }^{3}$, Elisabetta Renzoni ${ }^{4}$, \\ Simon L.F. Walsh ${ }^{1}$, David M. Hansell ${ }^{1}$ and Athol U. Wells ${ }^{4}$ \\ Affiliations: ${ }^{1}$ Department of Radiology, Royal Brompton Hospital, Royal Brompton and Harefield NHS \\ Foundation Trust, London, UK. ${ }^{2}$ Division of Radiology, Mayo Clinic Rochester, Rochester, Minnesota, USA. \\ ${ }^{3}$ Department of Physiology and Biomedical Engineering, Mayo Clinic Rochester, Rochester, Minnesota, USA. \\ ${ }^{4}$ Interstitial Lung Disease Unit, Royal Brompton Hospital, Royal Brompton and Harefield NHS Foundation \\ Trust, London, UK.
}

Correspondence: Joseph Jacob, 10 Wolsey Road, Northwood, Middlesex, HA6 2HW, UK. E-mail: joseph.jacobanhs.net

@ERSpublications

Emphysema in IPF has no effect on outcome beyond that explained by combined fibrosis and emphysema extents http://ow.ly/BtU430bUnIg

Cite this article as: Jacob J, Bartholmai BJ, Rajagopalan S, et al. Functional and prognostic effects when emphysema complicates idiopathic pulmonary fibrosis. Eur Respir J 2017; 50: 1700379 [https://doi.org/ 10.1183/13993003.00379-2017].

ABSTRACT This study aimed to investigate whether the combination of fibrosis and emphysema has a greater effect than the sum of its parts on functional indices and outcome in idiopathic pulmonary fibrosis (IPF), using visual and computer-based (CALIPER) computed tomography (CT) analysis.

Consecutive patients $(n=272)$ with a multidisciplinary IPF diagnosis had the extent of interstitial lung disease (ILD) scored visually and by CALIPER. Visually scored emphysema was subcategorised as isolated or mixed with fibrotic lung. The CT scores were evaluated against functional indices forced vital capacity (FVC), diffusing capacity of the lungs for carbon monoxide (DLCO), transfer coefficient of the lung for carbon monoxide (KCO), composite physiologic index (CPI)) and mortality.

The presence and extent of emphysema had no impact on survival. Results were maintained following correction for age, gender, smoking status and baseline severity using DLCO, and combined visual emphysema and ILD extent. Visual emphysema quantitation indicated that relative preservation of lung volumes (FVC) resulted from tractionally dilated airways within fibrotic lung, ventilating areas of admixed emphysema $(p<0.0001)$, with no independent effect on FVC from isolated emphysema. Conversely, only isolated emphysema $(\mathrm{p}<0.0001)$ reduced gas transfer $(D \mathrm{LCO})$.

There is no prognostic impact of emphysema in IPF, beyond that explained by the additive extents of both fibrosis and emphysema. With respect to the location of pulmonary fibrosis, emphysema distribution determines the functional effects of emphysema.

This article has supplementary material available from erj.ersjournals.com

Received: Jan 222017 | Accepted after revision: March 262017

Support statement: There is no funding source for the current study. The work was supported by the National Institute of Health Research Respiratory Disease Biomedical Research Unit at the Royal Brompton and Harefield NHS Foundation Trust and Imperial College London.

Conflict of interest: Disclosures can be found alongside this article at erj.ersjournals.com

Copyright OERS 2017 


\section{Introduction}

Patients with co-existing emphysema form a sizeable proportion of idiopathic pulmonary fibrosis (IPF) cohorts [1-4], a possible consequence of an association with smoking that is pertinent to both diseases $[5,6]$. A unique phenotype ascribed to patients with combined pulmonary fibrosis and emphysema (CPFE) was supported by the identification of poorer survival in patients with CPFE over those with isolated fibrosis [7-10]. The basis for a poor outcome is suggested to be partly related to an increased predisposition to the development of pulmonary hypertension (PHT) $[7,10,11]$. However, subsequent analyses of CPFE cohorts have provided conflicting data on the survival implications of emphysema co-existing with fibrosis $[4,12-15]$. Moreover, the question of whether the co-existence of pulmonary fibrosis and emphysema constitutes a discreet clinical syndrome remains unresolved $[16,17]$. Conflicting CPFE data have also led to the belief that CPFE should be viewed as a different disorder from IPF in terms of progression to death, and this in turn has caused some clinicians to have doubts about the use of anti-fibrotic therapy in CPFE.

A common constraint in the study of CPFE cohorts has been the limited CT quantification of the extent of emphysema, whether by visual or automated means. Simply ascertaining the presence or absence of emphysema restricts the precision with which a dose-effect of emphysema might be shown to influence survival. Similarly, the delineation and quantitation of emphysematous areas admixed within fibrotic lung, as opposed to emphysematous foci isolated from areas of fibrosis, has not been definitively examined to date. Nevertheless, quantifying emphysematous destruction within areas of fibrosis might inform and improve our understanding of the complex physiological effects that result from CPFE $[4,10,13,16,18]$.

In the current study, we therefore quantified the cumulative (morphological or functional) extents of emphysema and interstitial lung disease (ILD) in patients with IPF, utilising visual and computer-based CT analysis. Our primary aim was to identify whether a unique synergistic effect exists in IPF patients with emphysema, which results in a worsened outcome to a degree greater than that expected from the cumulative extents of ILD and emphysema. Secondarily, we wanted to investigate the functional impact of emphysema when it occurs both separately from and within fibrotic regions of the lung in IPF.

\section{Methods}

\section{Clinical data}

Retrospective analysis of an ILD database identified all consecutive, newly attending patients, who on re-analysis, received a multidisciplinary team (MDT) diagnosis of IPF according to published guidelines [19], over a $4 \frac{1}{2} 2$-year period (January 2007 to July 2011) $(n=283)$ [20]. Following publication of the INPULSIS study [21], all patients with a possible usual interstitial pneumonia (UIP) CT pattern were re-examined. Four patients were found to have a possible UIP pattern without traction bronchiectasis, whereas seven patients had CT findings inconsistent with a UIP diagnosis and no surgical lung biopsy. Accordingly, following exclusion of these 11 patients, the final study population comprised 272 IPF patients. A departmental, non-contrast, supine, volumetric CT was performed on all patients.

The CT, pulmonary function test protocols and CALIPER data processing steps are presented in the supplementary appendix. The study population has been described in two previous studies [20, 22].

\section{Ethics committee approval}

Approval for this retrospective study of clinically indicated CT and pulmonary function data was obtained from the Institutional Ethics Committee of the Royal Brompton Hospital and Mayo Clinic, and informed patient consent was not required.

\section{Computed tomographic evaluation}

CALIPER evaluation was performed as previously described (figure 1), and scores of ILD and extent of emphysema and pulmonary vessel volume (PVV) were considered in the analyses [22]. The CT scans were visually scored on a lobar basis with the extent of ILD, emphysema and honeycombing estimated to the nearest 5\%. Traction bronchiectasis [23] was assigned on a lobar basis with a categorical "severity" score, as previously described [22]. Calculations of the extent of admixed and isolated emphysema are explained in the supplementary appendix.

\section{Development of the modelling strategy}

In all analyses, adjustments were made for patient age, gender and smoking status. When analyses were re-examined unadjusted, cardinal results showed no change. Visual emphysema scores were found to be clearly superior to CALIPER emphysema scores, as described in the Supplementary Appendix. Therefore, in all subsequent analyses, visual emphysema scores were taken as the primary measure of emphysema. When the ILD score that best complimented visual emphysema was examined against our co-primary end-points (mortality and relationships to baseline DLCO (diffusing capacity of the lung for carbon monoxide)), the extent of CALIPER ILD was only marginally stronger than visual ILD scores at mortality prediction (table 6 
and supplementary table S3). Both ILD measures were therefore used in all subsequent analyses to ensure that conclusions made using CALIPER in the model were robust when visual ILD was substituted.

Three separate indices were used to adjust for baseline disease severity in all mortality analyses. The first index was functional, namely DLCO. The other two indices were morphological. To enable the evaluation of an extra-effect of CPFE on disease progression in excess of that expected from a combination of fibrosis and emphysema, the combined severity of emphysema and ILD was captured by summing visual emphysema extent scores with ILD extent scores, to create two indices of parenchymal damage: CILDemph=CALIPER ILD extent+visual emphysema extent; and VILDemph=visual ILD extent+visual emphysema extent. On linear regression analyses, there was a complete absence of collinearity between the presence of visual emphysema and CILDemph or VILDemph.

\section{Statistical analysis}

Data are given as the median or mean with standard deviations, depending on distributions, or the number of patients with percentages where appropriate. Differences between groups were evaluated using the Chi-squared test for categorical variables or a two-sample t-test for parametric continuous variables. Statistical significance was set at a value of $p<0.05$. Linear regression analyses were used to identify relationships between CT and functional indices, and PVV and emphysema. Univariate and multivariate Cox regression analyses were used to investigate variables predictive of mortality. Survival estimation was performed via the Kaplan-Meier method. Two-sample survival comparisons were performed using the log-rank test. Assumptions of linearity and proportional hazards were tested by visual inspection of Martingale residuals and scaled Schoenfeld residuals, and were satisfied. Statistical analyses were performed with the STATA software (version 12, StatCorp, College Station, TX, USA).

\section{Results}

\section{Demographic data}

Patients (272) diagnosed with IPF (supplementary figure S1) had vital status details completed on 268 out of 272 (99\%) cases, with four patients lost to follow up. Out of 272, 55 cases (20\%) had biopsy confirmation of the diagnosis. No differences in patient age or gender were identified between patients with and without emphysema. However, patients with emphysema were significantly more likely to develop lung cancer. Demographic details for patients with and without visually scored emphysema are presented in table 1 . The

TABLE 1 Characteristics of idiopathic pulmonary fibrosis patients with and without emphysema

\begin{tabular}{|c|c|c|c|}
\hline Variable & $\begin{array}{c}\text { Group } 1 \\
\text { No emphysema }\end{array}$ & $\begin{array}{c}\text { Group } 2 \\
\text { Visual emphysema }\end{array}$ & Group comparison \\
\hline Subjects & 167 & 105 & \\
\hline Median age years & 67 & 66 & $=0.05^{\#}$ \\
\hline Male/female $n / n$ & $126 / 41$ & $85 / 20$ & $=0.29^{\#}$ \\
\hline Survival (alive/dead) n/n & $49 / 118$ & $22 / 83$ & $=0.15^{\#}$ \\
\hline Never-/ever-smokers n/n & $82 / 85$ & $14 / 91$ & $<0.0001^{\#}$ \\
\hline Smoking history pack-years & $21.0 \pm 14.6(80)$ & $35.0 \pm 24.2(84)$ & $<0.0001^{\uparrow}$ \\
\hline Lung cancer prevalence $\%$ & 1.2 & 9.5 & $=0.001^{\#}$ \\
\hline FEV $1 \%$ predicted & $67.7 \pm 19.3(149)$ & $75.5 \pm 17.8(101)$ & $=0.002^{\Uparrow}$ \\
\hline FVC $\%$ predicted & $62.9 \pm 19.1(149)$ & $76.8 \pm 19.1(101)$ & $<0.0001^{\Uparrow}$ \\
\hline FEV $1 /$ FVC $\%$ predicted & $108.4 \pm 8.8(149)$ & $100.0 \pm 12.8(101)$ & $<0.0001^{\uparrow}$ \\
\hline DLco $\%$ predicted & $36.7 \pm 13.4(150)$ & $35.6 \pm 12.0(97)$ & $=0.52^{\text {ๆ }}$ \\
\hline Kco $\%$ predicted & $74.8 \pm 17.5(150)$ & $61.5 \pm 18.6(97)$ & $<0.0001^{\uparrow}$ \\
\hline TLC $\%$ predicted & $59.0 \pm 14.3(143)$ & $70.2 \pm 15.7(96)$ & $<0.0001^{\uparrow}$ \\
\hline CPI & $57.0 \pm 11.7(146)$ & $52.4 \pm 10.5(92)$ & $=0.001^{\Uparrow}$ \\
\hline CALIPER ILD extent \% & $29.3 \pm 18.5$ & $21.9 \pm 16.2$ & $=0.001^{\pi}$ \\
\hline Visual ILD extent \% & $49.3 \pm 17.7$ & $44.0 \pm 18.8$ & $=0.02^{\natural}$ \\
\hline Visual TxBx (scored as $0-18$ ) & $7.6 \pm 3.2$ & $6.5 \pm 3.2$ & $=0.008^{\text {I }}$ \\
\hline
\end{tabular}

Data are presented as mean $\pm \mathrm{SD}$, unless otherwise stated. Figures in brackets indicate the number of subjects for whom data was available. Significant differences between mean ranks of the two groups were calculated using the Chi-squared test for categorical independent variables $\left({ }^{\#}\right)$ and the t-test for continuous variables ("). FEV1: forced expiratory volume in $1 \mathrm{~s}$; FVC: forced vital capacity; DLco: diffusing capacity for carbon monoxide; Kco: carbon monoxide transfer coefficient; TLC: total lung capacity; CPI: composite physiologic index; ILD: interstitial lung disease; TxBx: traction bronchiectasis. 
TABLE 2 Relationships between pulmonary function indices and visual and CALIPER interstitial lung disease extent, and visual emphysema extent calculated using multivariate linear regression

\begin{tabular}{|c|c|c|c|c|c|c|}
\hline & $\begin{array}{l}\text { Pulmonary } \\
\text { function test }\end{array}$ & CT pattern & $\begin{array}{l}\text { Beta } \\
\text { coefficient }\end{array}$ & $95 \% \mathrm{Cl}$ & p-value & $\begin{array}{c}\text { Model } \\
\mathbf{R}^{2}\end{array}$ \\
\hline \multirow[t]{4}{*}{$\begin{array}{l}\text { Visual ILD and } \\
\text { emphysema evaluation }\end{array}$} & FVC & $\begin{array}{l}\text { Emphysema } \\
\text { ILD extent }\end{array}$ & $\begin{array}{c}0.48 \\
-0.49\end{array}$ & $\begin{array}{c}0.28-0.68 \\
-0.60--0.38\end{array}$ & $\begin{array}{l}<0.0001 \\
<0.0001\end{array}$ & 0.38 \\
\hline & DLCO & $\begin{array}{l}\text { Emphysema } \\
\text { ILD extent }\end{array}$ & $\begin{array}{l}-0.34 \\
-0.49\end{array}$ & $\begin{array}{l}-0.45--0.22 \\
-0.55--0.42\end{array}$ & $\begin{array}{l}<0.0001 \\
<0.0001\end{array}$ & 0.49 \\
\hline & Kco & $\begin{array}{l}\text { Emphysema } \\
\text { ILD extent }\end{array}$ & $\begin{array}{l}-0.88 \\
-0.42\end{array}$ & $\begin{array}{l}-1.06--0.70 \\
-0.53--0.42\end{array}$ & $\begin{array}{l}<0.0001 \\
<0.0001\end{array}$ & 0.44 \\
\hline & $\mathrm{CPI}$ & $\begin{array}{l}\text { Emphysema } \\
\text { ILD extent }\end{array}$ & $\begin{array}{l}-0.02 \\
0.44\end{array}$ & $\begin{array}{c}-0.12-0.09 \\
0.37-0.50\end{array}$ & $\begin{aligned} & =0.76 \\
& <0.0001\end{aligned}$ & 0.47 \\
\hline \multirow[t]{4}{*}{$\begin{array}{l}\text { CALIPER ILD and visual } \\
\text { emphysema evaluation }\end{array}$} & FVC & $\begin{array}{l}\text { Emphysema } \\
\text { ILD extent }\end{array}$ & $\begin{array}{c}0.31 \\
-0.67\end{array}$ & $\begin{array}{c}0.13-0.49 \\
-0.77--0.57\end{array}$ & $\begin{array}{l}=0.001 \\
<0.0001\end{array}$ & 0.52 \\
\hline & $D\llcorner C O$ & $\begin{array}{l}\text { Emphysema } \\
\text { ILD extent }\end{array}$ & $\begin{array}{l}-0.44 \\
-0.49\end{array}$ & $\begin{array}{l}-0.56--0.32 \\
-0.56--0.41\end{array}$ & $\begin{array}{l}=0.0001 \\
<0.0001\end{array}$ & 0.44 \\
\hline & Kco & $\begin{array}{l}\text { Emphysema } \\
\text { ILD extent }\end{array}$ & $\begin{array}{l}-0.91 \\
-0.23\end{array}$ & $\begin{array}{l}-1.10--0.71 \\
-0.35--0.11\end{array}$ & $\begin{array}{l}<0.0001 \\
=0.0002\end{array}$ & 0.33 \\
\hline & $\mathrm{CPI}$ & $\begin{array}{l}\text { Emphysema } \\
\text { ILD extent }\end{array}$ & $\begin{array}{l}0.09 \\
0.47\end{array}$ & $\begin{array}{c}-0.02-0.19 \\
0.41-0.54\end{array}$ & $\begin{array}{l}=0.10 \\
<0.0001\end{array}$ & 0.50 \\
\hline
\end{tabular}

The table is similar to table 3 , but instead of evaluating thresholds of emphysema, total emphysema extent was analysed in each model. All CT variables were calculated as a percentage of total lung volume. In all models, adjustments were made for patient age, gender and smoking status (never versus ever). CT: computed tomography; ILD: interstitial lung disease; FVC: forced vital capacity; DLCo: diffusing capacity for carbon monoxide; KCO: carbon monoxide transfer coefficient; CPI: composite physiological index.

single determination standard deviation for the visual emphysema scores was 4.80 (supplementary table S2) [24]. The kappa value for interobserver variation scoring of the presence of emphysema was 0.59 .

\section{Characterisation of the CPFE pulmonary function phenotype}

The presence of emphysema was associated with a relative preservation of lung volumes (FVC), while disproportionately reducing gas transfer ( $\mathrm{LCO})$ and the gas transfer coefficient (KCO). The results were maintained when emphysema was analysed as a continuous extent score (table 2), and when analysed at thresholds of $>0 \%,>5 \%$ and $>10 \%$ of visual emphysema (table 3). Linkages between the extent of ILD and emphysema, and DLCO are presented in supplementary table S3. The increase in FVC with emphysema in multivariate models reflects a relatively diminished FVC reduction for a given extent of fibrosis, and does not imply that emphysema causes an intrinsic rise in FVC.

No relationship was identified between the extent of total visual emphysema (or its isolated and admixed subcategories) and the extent of ILD scored either visually or by CALIPER (figure 1). The extent of both isolated and admixed emphysema were associated with obstructive functional indices, as determined by correlations of isolated $\left(\mathrm{R}^{2}=0.32, \mathrm{p}<0.0001\right)$ and admixed emphysema $\left(\mathrm{R}^{2}=0.14, \mathrm{p}<0.0001\right)$ with $F E V 1 / F V C$ ratios.

\section{Characterisation of functional effects of emphysema location}

Examination of the functional effects of isolated and admixed emphysema demonstrated that isolated emphysema was independently associated with lower DLCO and KCO, but had no impact on FVC or alveolar volume (VA) (table 4). Admixed emphysema was associated with preserved FVC and VA without any independent effects on DLCO, and opposing effects on VA and KCO (table 4).

\section{Effects of supervening emphysema on CT markers of fibrosis}

Evaluation of CT determinants of traction bronchiectasis demonstrated that emphysema had a negative correlation with traction bronchiectasis, whereas the extent of ILD and honeycombing demonstrated positive correlations (table 5). When isolated and admixed emphysema were substituted for the extent of total emphysema in the same model, only admixed emphysema demonstrated a strong negative linkage with traction bronchiectasis (table 5).

The pulmonary vascular volume has been previously shown to strongly predict outcome in patients with IPF [20], and consequently, links between PVV and emphysema were explored. After adjusting for the 
TABLE 3 Independent effects of CALIPER ILD extent and thresholds of visual emphysema on pulmonary function indices

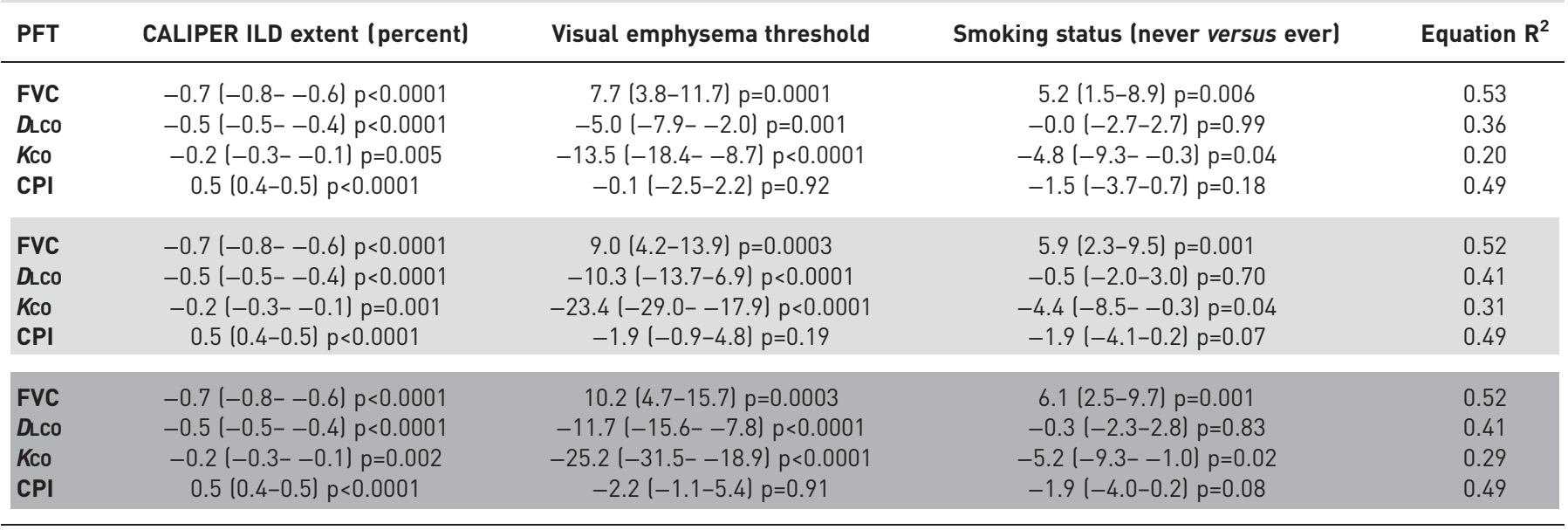

In all models, adjustments were made for patient age, gender and smoking status (never versus ever). The emphysema extent thresholds that were examined included $>0 \%$ visual emphysema (white), $>5 \%$ visual emphysema (light grey), $>10 \%$ visual emphysema (dark grey). CALIPER ILD extent was quantified as percentage of the lung; emphysema was categorised as presence above the relevant threshold; smokers were categorised as never- or ever-smokers. PFT: pulmonary function test; ILD: interstitial lung disease; FVC: forced vital capacity; FEV1: forced expiratory volume in one second; DLCO: diffusing capacity for carbon monoxide; KCO: carbon monoxide transfer coefficient; CPI: composite physiological index.

extent of ILD scored by CALIPER ( $\beta$ coefficient $=0.08,95 \%$ CI $0.08-0.09, p<0.0001$ ), the presence of emphysema ( $\beta$ coefficient $=-0.31,95 \%$ CI $-0.52--0.10, p=0.005)$ was independently associated with a minor $(6.0 \%)$ reduction in PVV (mean PVV=5.13\%) with a model $\mathrm{R}^{2}=0.75$.

\section{Impact of emphysema on survival in IPF}

With univariate analysis, when emphysema was evaluated as a continuous variable, a binary absence-presence score or a four-point categorical variable $(0=$ no emphysema, $1=0-5 \%$ emphysema, $2=5-$ $15 \%$ emphysema, $3=>15 \%$ emphysema), visually scored emphysema did not significantly predict mortality (table 6). The presence of emphysema did not alter outcome based on Kaplan-Meier analyses (figure 2a), with results being maintained in patients with severe disease (DLCO $<35 \%$ predicted) (figure $2 \mathrm{~b}$ ).

Emphysema is, on average, much less extensive than pulmonary fibrosis. For this reason, there is much less variation in the extent of emphysema than in the extent of fibrosis. Therefore, the prognostic value of variation in the extent of emphysema is overpowered/confounded in univariate analysis, because the highly variable extent of associated pulmonary fibrosis is not taken into account. On multivariate analysis however (table 6), visual emphysema (on a four-point scale) was independently predictive of mortality when analysed against and adjusted for the extent of CALIPER ILD. Neither the extent of isolated nor admixed emphysema was predictive of survival, following correction for the extent of global disease (using DLCO).

\section{Outcome adjusted for summed models of disease severity}

On univariate mortality analyses, the summed extent of CALIPER ILD and extent of visual emphysema (CILDemph), and the summed extent of visual ILD and extent of visual emphysema (VILDemph) both strongly predicted outcome (table 7). The CILDemph and VILDemph also demonstrated strong linkages with DLCO $\left(\mathrm{R}^{2}=0.43, \mathrm{p}<0.0001\right.$ and $\mathrm{R}^{2}=0.45, \mathrm{p}<0.0001$, respectively) (supplementary table $\mathrm{S} 3$ ).

When separately adjusting for disease severity (using CILDemph, VILDemph and DLCO), the presence of emphysema did not predict mortality independently (table 7), with results being maintained in patients with severe disease (DLCO $<35 \%$ predicted) (supplementary table S4).

As separate contributors to severity, both emphysema and fibrosis showed added linkage to mortality. However, once the total extent of disease was summed (as a combined fibrosis and emphysema score), it was immaterial (in terms of predicting mortality) whether the total extent of disease was due to pulmonary fibrosis alone, or a combination of pulmonary fibrosis and emphysema. In addition to an absence of evidence that CPFE was greater than the sum of its parts in predicting mortality, no link to more progressive disease (as judged by higher mortality for a given extent of disease) was identified in CPFE, following correction for the combined effects of emphysema and ILD. The multivariate analysis was 

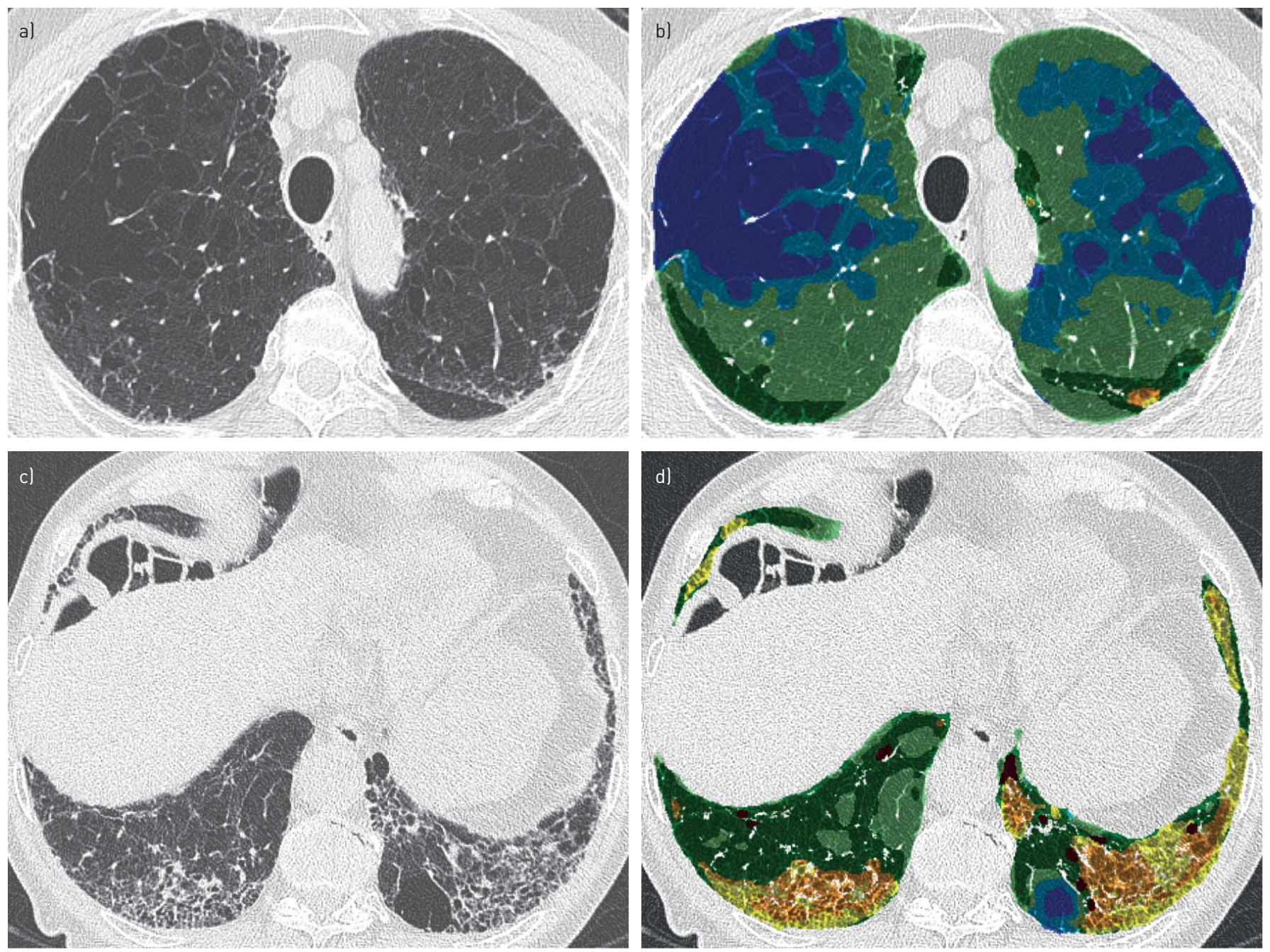

FIGURE 1 Axial computed tomography (CT) images and colour overlay images demonstrating quantitation of parenchymal patterns by CALIPER in a 71-year-old male ex-40-pack-year smoker diagnosed with idiopathic pulmonary fibrosis. CT images (a and c) demonstrate severe emphysema in the upper lobes, and fibrosis characterised primarily by a reticular pattern and traction bronchiectasis in the lower lobes, with an emphysematous bulla in the left lower lobe. On visual scoring, $40 \%$ of the lung was characterised as emphysema, whilst $31 \%$ was identified as interstitial lung disease. The CALIPER overlay images ( $b$ and $d$ ) outline emphysema (light and dark blue) in the upper lobes, quantified as $23 \%$ of lung volume. The sum of ground glass opacities (yellow), reticular pattern (orange) and honeycombing (brown) constitute the extent of total interstitial lung disease, which was quantified as $7.5 \%$ of the lung. CALIPER defines light and dark green areas as normal lung.

possible only because there was very little difference in the total extent of disease, regardless of whether emphysema was present, and no collinearity was present between the total extent score and the presence or absence of emphysema.

\section{Discussion}

Our study has shown that in a large consecutive IPF patient cohort, using a combination of visual analysis of emphysema and computer-based ILD quantitation, neither the presence nor the extent of emphysema impacts survival, following correction for baseline disease severity. For the first time, we have demonstrated the opposing effects on pulmonary volumes and gas transfer, when emphysema is primarily admixed within areas of fibrosis. Furthermore, we have demonstrated the inverse relationship between admixed emphysema and traction bronchiectasis, a validated CT marker of fibrosis.

In past evaluations of IPF patients with emphysema, analysis was concentrated on CPFE patients in whom emphysema was extensive. In the CPFE study by Cortin et al. [7], patient selection depended on clinician recollection of patients with concomitant emphysema and fibrosis. The possibility that a significant proportion of these cases had unusually extensive emphysema was suggested by the finding that 30 out of $61(49 \%)$ patients in that study [7] had an obstructive ventilatory defect ( $\mathrm{FEV} 1 / \mathrm{FVC}<70 \%$ predicted), compared to the current study, in which only two out of $101(2 \%)$ patients with emphysema demonstrated 
TABLE 4 Associations between subtypes of emphysema (percentage of the lung comprising emphysema separate from or admixed with fibrosis) and lung function indices, using multivariate linear regression

\begin{tabular}{|c|c|c|c|c|c|}
\hline PFT & CT pattern & $\begin{array}{c}\text { Beta } \\
\text { coefficient }\end{array}$ & $95 \% \mathrm{Cl}$ & p-value & Model $\mathbf{R}^{2}$ \\
\hline \multirow[t]{4}{*}{ FVC } & Isolated emphysema & 0.20 & $-0.22-0.63$ & $=0.35$ & 0.38 \\
\hline & Admixed emphysema & 0.73 & $0.36-1.11$ & $=0.0002$ & \\
\hline & ILD extent & -0.51 & $-0.62--0.39$ & $<0.0001$ & \\
\hline & Smoking status & 6.30 & $1.88-10.71$ & $=0.005$ & \\
\hline \multirow[t]{4}{*}{ D.co } & Isolated emphysema & -0.51 & $-0.75--0.27$ & $<0.0001$ & 0.50 \\
\hline & Admixed emphysema & -0.19 & $-0.40-0.03$ & $=0.09$ & \\
\hline & ILD extent & -0.50 & $-0.57--0.43$ & $<0.0001$ & \\
\hline & Smoking status & -0.11 & $-2.64-2.43$ & $=0.94$ & \\
\hline \multirow[t]{4}{*}{ Kco } & Isolated emphysema & -1.01 & $-1.39--0.63$ & $<0.0001$ & 0.44 \\
\hline & Admixed emphysema & -0.79 & $-1.12--0.45$ & $<0.0001$ & \\
\hline & ILD extent & -0.43 & $-0.54--0.32$ & $<0.0001$ & \\
\hline & Smoking status & -5.70 & $-9.68--1.41$ & $=0.005$ & \\
\hline \multirow[t]{4}{*}{$V A$} & Isolated emphysema & 0.15 & $-0.16-0.46$ & $=0.35$ & 0.38 \\
\hline & Admixed emphysema & 0.48 & $0.20-0.76$ & $=0.001$ & \\
\hline & ILD extent & -0.43 & $-0.52--0.35$ & $<0.0001$ & \\
\hline & Smoking status & 3.86 & $0.60-7.11$ & $=0.02$ & \\
\hline
\end{tabular}

All models were adjusted for extent of baseline visual interstitial lung disease (ILD) as a percentage of the lung, age, gender and smoking status (never versus ever). Extent of ILD and emphysema was calculated as a percentage of total lung volume. PFT: pulmonary function test; CT: computed tomography; FVC: forced vital capacity; $D\left\llcorner C 0\right.$ : diffusing capacity for carbon monoxide; KCO: carbon monoxide transfer coefficient; $V_{A}$ : alveolar volume.

an obstructive defect. Studies have also defined non-validated extent of emphysema thresholds (such as $>10 \%$ of the lung) in their CPFE inclusion criteria $[4,10]$. In the current study, only 35 out of $105(33 \%)$ CPFE patients had an extent of emphysema $>10 \%$ of the lung.

There is little evidence that cohort-wide estimations of the phenomenon of CPFE and its impact have been adequately studied. It would seem logical that once population-based assessments of emphysema have been made in IPF across the range of disease severity, as was the aim of the current study, questions relating to useful thresholds for the extent of emphysema could subsequently be addressed. Indeed, although emphysema thresholds per se, might have some value, greater importance likely lies in

TABLE 5 Visually and CALIPER scored CT determinants of traction bronchiectasis severity, evaluated using multivariate linear regression

\begin{tabular}{|c|c|c|c|c|c|}
\hline & СT Pattern & $\begin{array}{c}\text { Beta } \\
\text { coefficient }\end{array}$ & $95 \% \mathrm{Cl}$ & p-value & Model $\mathbf{R}^{2}$ \\
\hline \multirow[t]{3}{*}{ Visual } & Emphysema extent & -0.06 & $-0.09--0.03$ & $<0.0001$ & 0.51 \\
\hline & Honeycombing & 0.10 & $0.07-0.12$ & $<0.0001$ & \\
\hline & ILD extent & 0.06 & $0.05-0.08$ & $<0.0001$ & \\
\hline \multirow[t]{4}{*}{ Visual } & Isolated emphysema & -0.07 & $-0.13--0.01$ & $=0.02$ & 0.51 \\
\hline & Admixed emphysema & -0.06 & $-0.11--0.00$ & $=0.04$ & \\
\hline & Honeycombing & 0.10 & $0.07-0.12$ & $<0.0001$ & \\
\hline & ILD extent & 0.06 & $0.05-0.08$ & $<0.0001$ & \\
\hline \multirow[t]{3}{*}{ CALIPER } & Emphysema extent ${ }^{\#}$ & -0.10 & $-0.14--0.07$ & $<0.0001$ & 0.36 \\
\hline & Honeycombing & 0.84 & $0.64-1.05$ & $<0.0001$ & \\
\hline & ILD extent & 0.06 & $0.04-0.07$ & $<0.0001$ & \\
\hline
\end{tabular}

Extent of visually scored emphysema, ILD and honeycombing, expressed as a percentage of the lung were all independently associated with severity of traction bronchiectasis. All models were adjusted for patient age, gender and smoking status (never versus ever). ": Emphysema was only quantified visually. CT: computed tomography; ILD: interstitial lung disease. 

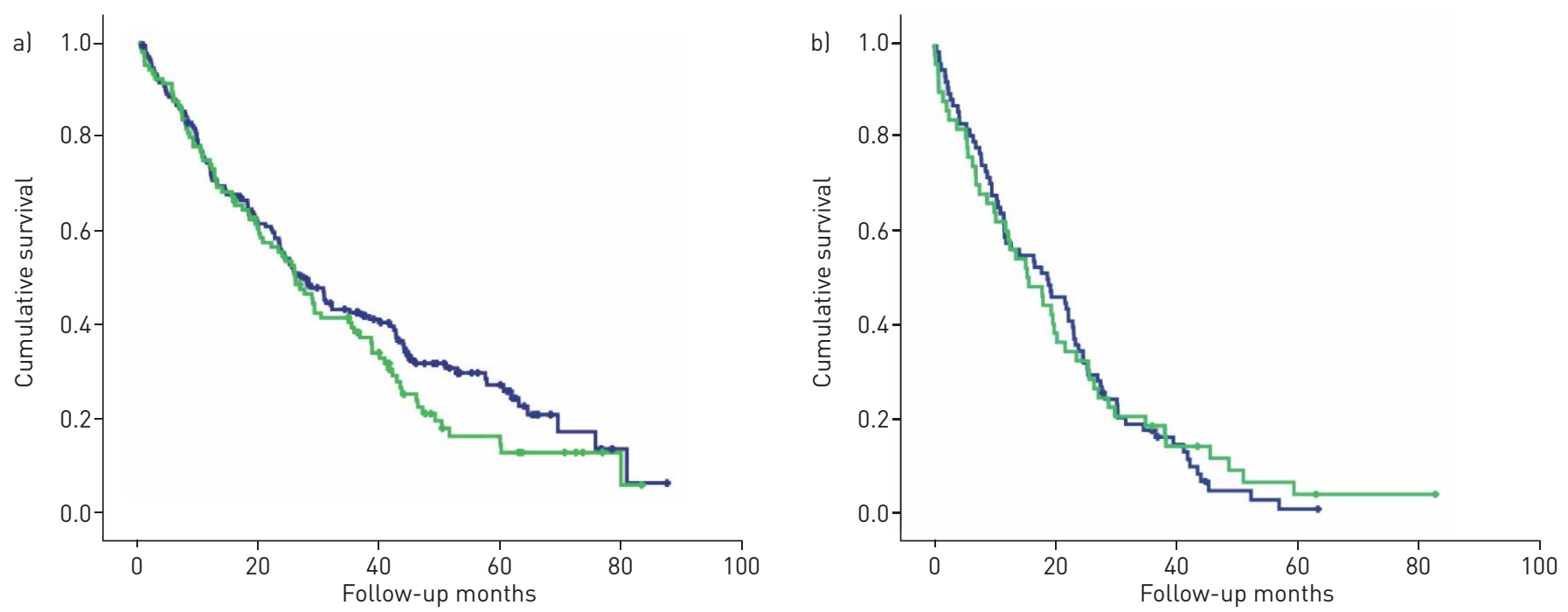

FIGURE 2 a) Kaplan-Meier survival curves were not found to be significantly different in outcome between idiopathic pulmonary fibrosis (IPF) patients without visually scored emphysema on computed tomography (CT) (blue; $n=167$, restricted mean survival=36.5 \pm 2.3 ), and IPF patients with emphysema scored visually on CT (green; $n=105$, restricted mean survival $=32.0 \pm 2.5$ ). Log rank test $p=0.20$. b) Kaplan-Meier survival curves were not found to be significantly different in outcome between IPF patients with a baseline diffusing capacity of the lungs for carbon monoxide ( $D$ Lco) $<35 \%$ predicted without visually scored emphysema on CT (blue; $n=79$, restricted mean survival $=20.9 \pm 1.8$ ), and IPF patients with emphysema scored visually on CT (green; $n=51$, restricted mean survival=21.8 \pm 2.9 ). Log rank test $p=0.84$.

delineation of the predominant pathology in any single patient, namely deciding whether emphysema is more extensive than fibrosis in the lungs. In this regard, technological advances used in the current study, that were not available to prior investigations describing CPFE, might help to improve characterisation of the extent of both emphysema and fibrosis. In addition to the utilisation of automated CT quantitation, which can improve on visual CT evaluation of ILD extent [20,22], we utilised volumetric CT acquisitions that allow the visual quantitation of emphysema and visual and computer-based quantitation of ILD across the entire lung volume, rather than at sampled interspaced levels.

In line with previous reports, in our study, IPF when co-existing with emphysema did not progress at a faster trajectory $[4,12]$. In contrast, the prognosis in CPFE was more heavily aligned with baseline disease severity. The continued discordance in fundamental outcome measures, such as mortality, between CPFE reports $[4,7,10,12]$ supports the pressing need for a definition, which does not currently exist, of what

TABLE 6 Univariate CALIPER and visually derived CT variables and pulmonary function indices predictive of mortality using Cox proportional hazards regression models

\begin{tabular}{lccc} 
Baseline variables & Hazard ratio & p-value & $\mathbf{9 5 \%} \mathbf{~ C l}$ \\
\hline Visual emphysema (continuous) & 1.01 & $=0.18$ & $1.00-1.02$ \\
Visual emphysema (categorical) & 1.10 & $=0.17$ & $0.96-1.25$ \\
Visual emphysema (presence) & 1.18 & $=0.26$ & $0.89-1.56$ \\
Visual ILD extent & 1.03 & $<0.0001$ & $1.02-1.04$ \\
CALIPER emphysema & 1.00 & $=0.84$ & $0.98-1.03$ \\
CALIPER ILD extent & 1.03 & $<0.0001$ & $1.03-1.04$ \\
CILDemph & 1.03 & $<0.0001$ & $1.03-1.04$ \\
VILDemph & 1.02 & $<0.0001$ & $1.02-1.03$ \\
FVC & 1.07 & $<0.0001$ & $1.06-1.08$ \\
DLCO & 1.04 & $<0.0001$ & $1.03-1.05$ \\
CPI & 1.07 & $<0.0001$ & $1.05-1.08$ \\
\hline
\end{tabular}

Visual emphysema was scored as a continuous variable, as a 4-point categorical variable $10=$ no emphysema, $1=0-5 \%$ emphysema, $2=5-15 \%$ emphysema, $3=>15 \%$ emphysemal and as a binary, absence-presence variable. ILD: interstitial lung disease; CILDemph: summed total of extent of CALIPER ILD and extent of visual emphysema; VILDemph: summed total of extent of visual ILD and extent of visual emphysema; FVC: forced vital capacity; DLCO: diffusing capacity for carbon monoxide, CPI: composite physiologic index. 


\begin{tabular}{|c|c|c|c|c|c|}
\hline \multirow[t]{2}{*}{ Patient subset } & \multirow[t]{2}{*}{ Baseline severity and emphysema models } & \multirow[t]{2}{*}{ Hazard ratio } & \multirow[t]{2}{*}{ p-value } & \multicolumn{2}{|c|}{$95 \% \mathrm{Cl}$} \\
\hline & & & & Lower & Upper \\
\hline \multirow[t]{4}{*}{ All patients } & Visual Emphysema categorical & 1.23 & $=0.006$ & 1.06 & 1.43 \\
\hline & CALIPER ILD extent & 1.04 & $<0.0001$ & 1.03 & 1.04 \\
\hline & Visual Emphysema categorical & 1.13 & $=0.09$ & 1.08 & 1.31 \\
\hline & Visual ILD extent & 1.03 & $<0.0001$ & 1.02 & 1.04 \\
\hline \multirow[t]{2}{*}{ Model 1} & CILDemph & 1.03 & $<0.0001$ & 1.03 & 1.04 \\
\hline & Visual Emphysema presence & 0.93 & $=0.67$ & 0.68 & 1.29 \\
\hline \multirow[t]{2}{*}{ Model 2} & VILDemph & 1.03 & $<0.0001$ & 1.02 & 1.03 \\
\hline & Visual Emphysema presence & 0.94 & $=0.73$ & 0.68 & 1.30 \\
\hline \multirow[t]{2}{*}{ Model 3} & DLco & 0.94 & $<0.0001$ & 0.93 & 0.95 \\
\hline & Visual Emphysema presence & 0.98 & $=0.93$ & 0.71 & 1.37 \\
\hline \multirow[t]{2}{*}{ Model 1} & CILDemph & 1.03 & $<0.0001$ & 1.03 & 1.04 \\
\hline & Visual Emphysema categorical & 0.91 & $=0.20$ & 0.78 & 1.05 \\
\hline \multirow[t]{2}{*}{ Model 2} & VILDemph & 1.03 & $<0.0001$ & 1.02 & 1.04 \\
\hline & Visual Emphysema categorical & 0.88 & $=0.11$ & 0.75 & 1.03 \\
\hline \multirow[t]{2}{*}{ Model 3} & $D\llcorner\mathrm{LCO}$ & 0.94 & $<0.0001$ & 0.93 & 0.95 \\
\hline & Visual Emphysema categorical & 0.97 & $=0.68$ & 0.83 & 1.13 \\
\hline
\end{tabular}

In an examination of all patients in the cohort ( $n=272$ ), extent of baseline ILD scored using CALIPER and visual assessment were separately evaluated in models against extent of visual emphysema. Visual emphysema was scored as a 4-point categorical variable $10=$ no emphysema, $1=0-5 \%$ emphysema, $2=5-15 \%$ emphysema, $3=>15 \%$ emphysema). In a separate sub-analysis of patients with severe/end stage disease ( $n=130 / 272$ ), two morphological measures of severity of baseline disease were analysed. The first measure represented the combination of visual emphysema scores with CALIPER-derived ILD extent: CILDemph (Model 1), and the second measure represented the combination of visual emphysema scores with extent of visually-derived ILD: VILDemph (Model 2). A third measure of baseline disease severity was a functional severity measure: DLCo (Model 3). To evaluate whether the presence of emphysema had any impact on outcome after adjustment for severity of total baseline disease, all three models were separately evaluated alongside the presence of emphysema and the four-point categorical emphysema score. All models were adjusted for patient age, gender and smoking status (never versus ever). ILD: interstitial lung disease; CILDemph: summed total of extent of CALIPER ILD and extent of visual emphysema; VILDemph: summed total of extent of visual ILD and extent of visual emphysema; DLCO: diffusing capacity for carbon monoxide.

specifically constitutes CPFE. An international initiative to agree on a CPFE definition is clearly warranted, to curtail the real danger of an impossibility to integrate future studies in CPFE.

Our findings provide further confirmation of the unique functional profile that occurs when emphysema co-exists with IPF $[4,7,10,13,18]$. Emphysema preserves lung volumes, limiting the utility of FVC to act as an index to adjust for the severity of baseline disease in CPFE. The composite physiologic index (CPI) is negated in CPFE, as it only measures the functional impact of fibrosis and not emphysema. The DLCO represents the cardinal functional index in CPFE patients, as it reflects the contribution of both interstitial fibrosis and emphysema to the reduction of gas exchange. The strength of DLCO as a measure of disease severity in CPFE was confirmed by the strikingly similar results in our study, when DLCO and indices that reflect cumulative pulmonary damage (summed extent of visual or CALIPER ILD and visual emphysema) were used to control for baseline disease severity. Furthermore, the similarities in analyses between morphological scores and DLCO validated our chosen methodological approach of combining quantitative and visual CT measures.

The clinical observations of the current study were made more robust by the utilisation of independent methods of scoring the extent of ILD. Integrating automated and visual analysis is valuable; for example in our analyses, we selected variables for which the use of CALIPER is an advantage (precision in delineating ILD extent), and variables for which expert visual judgements are required (distinguishing admixed 
emphysema from honeycomb cysts). Had a strategy of utilising only automated scores been adopted in the current study, the distinct functional effects of admixed emphysema would not have been discovered. Although subjective and increasingly, automated scoring have their proponents, the best model might be a combination of both modalities. A recent report by ARAKI et al. [25] in a large Framingham Heart study cohort remarkably highlighted the constraints that can arise when relying solely on an automated method of quantitation of interstitial lung abnormalities (ILAs). The subtle differentiation of minor fibrotic changes, as seen in respiratory bronchiolitis, from abnormalities that are more compatible with early IPF, such as sub-pleural reticular abnormalities, are not yet possible with automated systems, and such analyses can be enhanced by the addition of visual characterisation of ILAs.

Most studies that have evaluated emphysema in IPF have been hampered, as previously described, by limitations in CT quantitation of the extent of fibrosis and emphysema [4, 10, 15, 26]. Automated quantitation studies have also been hampered by small sample sizes $[8,27-30]$ and the challenges of distinguishing emphysema from honeycomb cysts or traction bronchiectasis. The challenge of separating emphysema from honeycombing by a computer tool remains unmet, and is the reason for the reliance on visual emphysema scores for emphysema quantitation and characterisation in the analyses of the current study.

Uniquely, we have identified that pure and admixed emphysema are associated with distinct functional consequences. Admixed emphysema preserved lung volumes, including FVC and VA, in contrast to isolated emphysema. Emphysema is typically associated with air-trapping, as a result of airway narrowing and collapse on expiration, as bullous spaces fail to deflate [31]. In areas of fibrosis however, contraction of the interstitial connective tissue framework can pull small airways open [32], which is visible on CT as traction bronchiectasis [33], to allow the ventilation of emphysematous airspaces with consequent preservation of FVC and VA. In our analyses, a reduction in DLCO values was primarily related to the extent of ILD, and not to admixed emphysema. The destruction of capillary beds in areas of isolated emphysema is thought to inhibit gas transfer (DLCO), by reducing blood volume within the lungs. However, in areas of admixed emphysema, vascular destruction might be a consequence of both fibrotic and emphysematous processes. Emphysema had a greater impact on KCO than the extent of ILD scored either visually or by CALIPER, which is consistent with previous findings [1]. However, preservation of alveolar volume by admixed emphysema did influence the gas transfer coefficient, which is synonymous with $D$ LCO/VA.

The severity of traction bronchiectasis, a cardinal morphological measure of disease severity in IPF [20, 34], was found to be inversely related to the extent of emphysema (admixed) in the present study, in keeping with the findings of a previous report [35]. Although traction bronchiectasis enables bullae to remain ventilated, the emphysema-induced parenchymal damage that precedes interstitial fibrosis might limit the degree to which airways can be pulled open by a contracted and fibrosed connective tissue scaffold, when compared to areas of fibrosis without admixed emphysema. The relative reduction in the extent of ILD in CPFE patients compared to IPF patients in the current study, is consistent with previous results [4], and might be reflective of earlier recognition of dyspnoea during the course of IPF disease in a patient, as emphysema reduces the functional reserve.

The PVV was shown to be reduced by $6 \%$ in IPF patients with emphysema and this might suggest alveolar and capillary destruction in emphysema [36], resulting in reduced vascularity, quantified by CALIPER within regions of emphysematous lung. It has also been suggested that the high negative intrathoracic pressures required for inspiration in patients with fibrosis might pull on the walls of the pulmonary vessels, and thereby result in an increase in the PVV. Consequently, when emphysema co-exists with fibrosis, a relative reduction in intrathoracic pressures might result in a slight reduction in the PVV, when compared to patients with fibrosis alone.

In our study, when correcting for the visual extent of emphysema, and at thresholds of $>5 \%$ and $>10 \%$ visual emphysema, smoking status had an independent effect on impairment of pulmonary function. Specifically, a positive smoking history elevated FVC and reduced KCO by $6 \%$, which is very similar to the findings noted in a contemporaneous study evaluating the effects of smoking and emphysema in scleroderma [37]. However, a report by WeLls et al. [1] demonstrated that after correcting for the presence of emphysema, smoking status had no independent effect on impairment of pulmonary function. Although a smoking history would appear at first to be at odds with FVC elevation and KCO inhibition, these effects might simply reflect a link with emphysema that is secondary to smoking. As visual evaluation of a CT might only capture a proportion of the emphysema present within the lungs, the emergence of a statistically significant smoking history might indicate that the extent of emphysema has been underestimated by visual scores; a phenomenon that appears greatest at extremes of the extent of emphysema. 
There were some limitations in the current study. Histopathological proof of an IPF diagnosis was lacking in the majority of patients; however, all cases were reviewed according to current diagnostic and treatment guidelines [19] in what is now the accepted standard of an MDT setting. Distinguishing admixed emphysema from honeycomb cysts is associated with poor interobserver agreement [38], and might have limited the reliability with which the extent of emphysema was visually characterised. However, the negative correlations between the admixed emphysema scores and the FEV1/FVC ratio indicate that for the most part, honeycomb cysts were not being misclassified as emphysema.

In conclusion, we have demonstrated that baseline disease severity specifically determines outcome in a patient with IPF, and that co-existing emphysema does not have any additional negative impact on outcome. We have demonstrated that DLCO, by capturing the effects of both interstitial damage and emphysema, is the optimal measure of disease severity when emphysema co-exists with fibrosis. Our study has also highlighted the physiological subtleties that develop when emphysema is both isolated from and admixed within areas of fibrosis.

\section{Acknowledgements}

Author contributions: J. Jacob, M. Kokosi, T. Maher, A. Nair, E. Renzoni, S.L.F. Walsh, A.U. Wells, D.M. Hansell were involved in either the acquisition, analysis or interpretation of data for the study.

J. Jacob, A.U. Wells and D.M. Hansell were also involved in the conception and design of the study.

B.J. Bartholmai, R. Karwoski and S. Rajagopalan invented and developed CALIPER. They were also involved in processing the raw CT scans and generation of figures, but were not involved in the analysis or interpretation of data in the study.

J. Jacob had full access to all the data in the study and had final responsibility for the decision to submit for publication.

\section{References}

1 Wells AU, King AD, Rubens MB, et al. Lone cryptogenic fibrosing alveolitis: a functional-morphologic correlation based on extent of disease on thin-section computed tomography. Am J Respir Crit Care Med 1997; 155: 1367-1375.

2 Wells AU, Desai SR, Rubens MB, et al. Idiopathic pulmonary fibrosis: a composite physiologic index derived from disease extent observed by computed tomography. Am J Respir Crit Care Med 2003; 167: 962-969.

3 Mitchell PD, Das JP, Murphy DJ, et al. Idiopathic pulmonary fibrosis with emphysema: evidence of synergy among emphysema and idiopathic pulmonary fibrosis in smokers. Respir Care 2015; 60: 259-268.

4 Ryerson C, Hartman T, Elicker B, et al. Clinical features and outcomes in combined pulmonary fibrosis and emphysema in idiopathic pulmonary fibrosis. Chest 2013; 144: 234-240.

5 Papiris SA, Triantafillidou C, Manali ED, et al. Combined pulmonary fibrosis and emphysema. Expert Rev Respir Med 2013; 7: 19-32.

6 Tasaka S, Mizoguchi K, Funatsu Y, et al. Cytokine profile of bronchoalveolar lavage fluid in patients with combined pulmonary fibrosis and emphysema. Respirology 2012; 17: 814-820.

7 Cottin V, Nunes H, Brillet PY, et al. Combined pulmonary fibrosis and emphysema: a distinct underrecognised entity. Eur Respir J 2005; 26: 586-593.

8 Lee $\mathrm{CH}$, Kim HJ, Park CM, et al. The impact of combined pulmonary fibrosis and emphysema on mortality. Int $\mathrm{J}$ Tuberc Lung Dis 2011; 15: 1111-1116.

9 Sugino K, Ishida F, Kikuchi N, et al. Comparison of clinical characteristics and prognostic factors of combined pulmonary fibrosis and emphysema versus idiopathic pulmonary fibrosis alone. Respirology 2014; 19: 239-245.

10 Mejía M, Carrillo G, Rojas-Serrano J, et al. Idiopathic pulmonary fibrosis and emphysema: Decreased survival associated with severe pulmonary arterial hypertension. Chest 2009; 136: 10-15.

11 Cottin V, Le PJ, Prevot G, et al. Pulmonary hypertension in patients with combined pulmonary fibrosis and emphysema syndrome. Eur Respir J 2010; 35: 105-111.

12 Jankowich MD, Rounds S. Combined pulmonary fibrosis and emphysema alters physiology but has similar mortality to pulmonary fibrosis without emphysema. Lung 2010; 188: 365-373.

13 Bodlet A, Maury G, Jamart J, et al. Influence of radiological emphysema on lung function test in idiopathic pulmonary fibrosis. Respir Med 107: 1781-1788.

14 Kurashima K, Takayanagi N, Tsuchiya N, et al. The effect of emphysema on lung function and survival in patients with idiopathic pulmonary fibrosis. Respirology 2010; 15: 843-848.

15 Todd N, Jeudy J, Lavania S, et al. Centrilobular emphysema combined with pulmonary fibrosis results in improved survival. Fibrogenesis Tissue Repair 2011; 4: 6.

16 Cottin V, Cordier JF. Combined pulmonary fibrosis and emphysema: an experimental and clinically relevant phenotype. Am J Respir Crit Care Med 2005; 172: 1605-1606.

17 Cottin V, Cordier J-F. The syndrome of combined pulmonary fibrosis and emphysema. Chest 2009; 136: 1-2.

18 Mura M, Zompatori M, Pacilli AM, et al. The presence of emphysema further impairs physiologic function in patients with idiopathic pulmonary fibrosis. Respir Care 2006; 51: 257-265.

19 Raghu G, Collard HR, Egan JJ, et al. An official ATS/ERS/JRS/ALAT statement: idiopathic pulmonary fibrosis: evidence-based guidelines for diagnosis and management. Am J Respir Crit Care Med 2011; 183: 788-824.

20 Jacob J, Bartholmai B, Rajagopalan S, et al. Mortality prediction in idiopathic pulmonary fibrosis: evaluation of automated computer tomographic analysis with conventional severity measures. Eur Respir J 2017; 49: 1601011.

21 Richeldi L, du Bois RM, Raghu G, et al. Efficacy and safety of nintedanib in idiopathic pulmonary fibrosis. $N$ Engl J Med 2014; 370: 2071-2082.

22 Jacob J, Bartholmai B, Rajagopalan S, et al. Automated quantitative CT versus visual CT scoring in idiopathic pulmonary fibrosis: validation against pulmonary function. J Thorac Imaging 2016; 31: 304-311. 
23 Hansell DM, Bankier AA, MacMahon H, et al. Fleischner Society: glossary of terms for thoracic imaging. Radiology 2008; 246: 697-722.

24 Chinn S. Statistics in respiratory medicine. 2. Repeatability and method comparison. Thorax 1991; 46: 454-456.

25 Araki T, Putman RK, Hatabu H, et al. Development and progression of interstitial lung abnormalities in the Framingham Heart Study. Am J Respir Crit Care Med 2016; 194: 1514-1522.

26 Schmidt SL, Nambiar AM, Tayob N, et al. Pulmonary function measures predict mortality differently in IPF versus combined pulmonary fibrosis and emphysema. Eur Respir J 2011; 38: 176-183.

27 Ando K, Sekiya M, Tobino K, et al. Relationship between quantitative CT metrics and pulmonary function in combined pulmonary fibrosis and emphysema. Lung 2013; 191: 585-591.

28 Tanizawa K, Handa T, Nagai S, et al. Clinical impact of high-attenuation and cystic areas on computed tomography in fibrotic idiopathic interstitial pneumonias. BMC Pulm Med 2015; 15: 74

29 Maldonado F, Moua T, Rajagopalan S, et al. Automated quantification of radiological patterns predicts survival in idiopathic pulmonary fibrosis. Eur Respir J 2014; 43: 204-212.

30 Matsuoka S, Yamashiro T, Matsushita S, et al. Quantitative CT evaluation in patients with combined pulmonary fibrosis and emphysema: correlation with pulmonary function. Acad Radiol 2015; 22: 626-631.

31 Crystal RG, Fulmer JD, Roberts WC, et al. Idiopathic pulmonary fibrosis: clinical, histologic, radiographic, physiologic, scintigraphic, cytologic and biochemical aspects. Ann Intern Med 1976; 85: 769-788.

32 Strickland NH, Hughes JM, Hart DA, et al. Cause of regional ventilation-perfusion mismatching in patients with idiopathic pulmonary fibrosis: a combined CT and scintigraphic study. AJR Am J Roentgenol 1993; 161: 719-725.

33 Remy-Jardin M, Giraud F, Remy J, et al. Importance of ground-glass attenuation in chronic diffuse infiltrative lung disease: pathologic-CT correlation. Radiology 1993; 189: 693-698.

34 Sumikawa $\mathrm{H}$, Johkoh T, Colby TV, et al. Computed tomography findings in pathological usual interstitial pneumonia: relationship to survival. Am J Respir Crit Care Med 2008; 177: 433-439.

35 Desai SR, Wells AU, Rubens MB, et al. Traction bronchiectasis in cryptogenic fibrosing alveolitis: associated computed tomographic features and physiological significance. Eur Radiol 2003; 13: 1801-1808.

36 Snider GL, Kleinerman J, Thurlbeck WM, et al. The definition of emphysema. Report of a National Heart, Lung, and Blood Institute, Division of Lung Diseases workshop. Am Rev Respir Dis 1985; 132: 182-185.

37 Antoniou KM, Margaritopoulos GA, Goh NS, et al. Combined pulmonary fibrosis and emphysema in scleroderma lung disease has a major confounding effect on lung physiology and screening for pulmonary hypertension. Arthritis Rheumatol 2015; 68: 1004-1012.

38 Watadani T, Sakai F, Johkoh T, et al. Interobserver variability in the CT assessment of honeycombing in the lungs. Radiology 2013; 266: 936-944. 